\title{
On the Kissing Numbers of Some Special Convex Bodies*
}

\author{
D. G. Larman ${ }^{1}$ and C. Zong ${ }^{1,2}$ \\ ${ }^{1}$ Department of Mathematics, University College London, \\ Gower Street, London WC1E 6BT, England \\ ros@math.ucl.ac.uk \\ ${ }^{2}$ Institute of Mathematics, The Chinese Acadamy of Sciences, \\ Beijing 100080, People's Republic of China \\ cmzong@math08.math.ac.cn
}

\begin{abstract}
In this note the kissing numbers of octahedra, rhombic dodecahedra and elongated octahedra are determined. In high dimensions, an exponential lower bound for the kissing numbers of superballs is achieved.
\end{abstract}

\section{Introduction}

Let $K$ be an $n$-dimensional convex body. As usual, we denote the translative kissing number and the lattice kissing number of $K$ by $N(K)$ and $N^{*}(K)$, respectively. In other words, $N(K)$ is the maximal number of nonoverlapping translates of $K$ which can be brought into contact with $K$, and $N^{*}(K)$ is the similar number when the translates are taken from a lattice packing of $K$.

To determine the values of $N(K)$ and $N^{*}(K)$ for a convex body $K$ are important and difficult problems in the study of packings. These numbers, especially for balls, have been studied by many well-known mathematicians such as Newton, Minkowski, Hadwiger, van der Waerden, Shannon, Leech, Gruber, Hlawka, Kabatjanski, Levenštein, Odlyzko, Rankin, Rogers, Sloane, Watson, Wyner and many others. For details refer to [1]-[3] and [11].

In this note we determine the kissing numbers of octahedra, rhombic dodecahedra and elongated octahedra. In fact, besides balls and cylinders, they are the only convex bodies whose kissing numbers are exactly known. In Section 4 the $n$-dimensional superballs are considered.

\footnotetext{
* The work of the second author was supported by a research fellowship of The Royal Society.
} 
Let $\delta^{C}$ be the Minkowski-metric in $R^{n}$ given by a centrally symmetric convex body $C$. In other words, denote by $C(\mathbf{z})$ the boundary point of $C$ at direction $\mathbf{z}$,

$$
\delta^{C}(\mathbf{x}, \mathbf{y})=\left\{\begin{array}{lll}
\frac{\|\mathbf{x}-\mathbf{y}\|}{\|C(\mathbf{x}-\mathbf{y})\|} & \text { if } & \mathbf{x} \neq \mathbf{y}, \\
0 & \text { if } & \mathbf{x}=\mathbf{y},
\end{array}\right.
$$

where $\|\cdot\|$ indicates the Euclidean norm (see [5]). To determine the kissing numbers of octahedra, rhombic dodecahedra and elongated octahedra, the following lemma is frequently demanded.

Lemma 1 [10]. If the boundary $\partial(C)$ of $C$ can be divided into $m$ subsets $X_{1}, X_{2}$, $\ldots, X_{m}$ such that $\delta^{C}(\mathbf{x}, \mathbf{y})<1$ whenever both $\mathbf{x}$ and $\mathbf{y}$ belong to the same subset, then $N(C) \leq m$. More precisely, if $Z=\left\{\mathbf{z}_{1}, \mathbf{z}_{2}, \ldots, \mathbf{z}_{l}\right\}$ is a set of points such that $C+\{2 Z\} \cup\{\mathbf{o}\}$ forms a kissing configuration of $C$, then any subset $X_{i}$ contains at most one point of $Z$.

For convenience, we denote by $(\mathbf{x y} \cdots \mathbf{z})$ an open (relatively) polygonal arc, by $(\mathbf{x y} \cdots \mathbf{z}]$ a half open and half closed one, and by $[\mathbf{x y} \cdots \mathbf{z}]$ a closed one.

\section{Octahedra}

Theorem 1. Let $O$ be an octahedron, then $N(O)=N^{*}(O)=18$.

Proof. For convenience, we take

$$
O=\left\{\mathbf{x} \in R^{3}:\left|x^{1}\right|+\left|x^{2}\right|+\left|x^{3}\right| \leq 1\right\},
$$

and let $\Lambda$ be the lattice generated by $\mathbf{a}=(1,1,0), \mathbf{b}=(1,-1,0)$ and $\mathbf{c}=(1,0,1)$. It is easy to see that $O+\Lambda$ is a lattice packing with density $\frac{2}{3}$, in which every octahedron touches 18 others. So that

$$
N^{*}(O) \geq 18
$$

Let $\mathbf{x}_{1,1}, \mathbf{x}_{2,2}, \ldots, \mathbf{x}_{6,6}$ be the six vertices of $O$ indicated by Fig. 1 , let $\mathbf{x}_{i, j}$ be the midpoint of $\mathbf{x}_{i, i} \mathbf{x}_{j, j}$, and let $\mathbf{x}_{i^{\prime}, j, k}, \mathbf{x}_{i, j^{\prime}, k}$ and $\mathbf{x}_{i, j, k^{\prime}}$ be points indicated by Fig. 2, where $\left\|\mathbf{u} \mathbf{x}_{i, i}\right\|=\frac{1}{4}\left\|\mathbf{x}_{i, i} \mathbf{x}_{j, j}\right\|$. Writing

$$
Y_{i, j}=\operatorname{rint}\left(\left(\frac{1}{2} \mathbf{x}_{i, j}+\frac{1}{2} O\right) \cap \partial(O)\right),
$$

where $\operatorname{rint}(X)$ indicates the relative interior of $X$, and defining

$$
\begin{gathered}
X_{i, i}=Y_{i, i}, \quad i=1,2, \ldots, 6, \\
X_{1,2}=Y_{1,2} \cup\left\{\mathbf{x}_{1,2^{\prime}, 6}, \mathbf{x}_{1,2^{\prime}, 5}\right\}, \quad X_{1,4}=Y_{1,4} \cup\left\{\mathbf{x}_{1^{\prime}, 4,6}, \mathbf{x}_{1^{\prime}, 4,5}\right\}, \\
X_{1,5}=Y_{1,5} \cup\left\{\mathbf{x}_{1^{\prime}, 2,5}, \mathbf{x}_{1,2,5^{\prime}}\right\},
\end{gathered}
$$




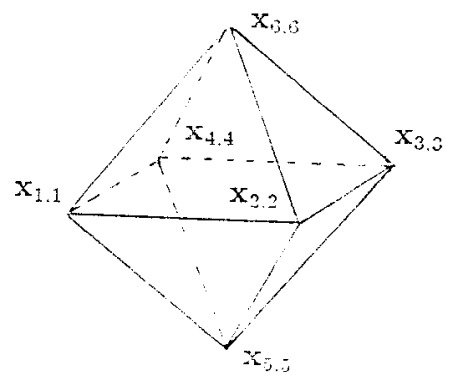

Fig. 1

$$
\begin{array}{ll}
X_{2,3}=Y_{2,3} \cup\left\{\mathbf{x}_{2,3^{\prime}, 6}, \mathbf{x}_{2,3^{\prime}, 5}\right\}, & X_{2,5}=Y_{2,5} \cup\left\{\mathbf{x}_{2^{\prime}, 3,5}, \mathbf{x}_{2,3,5^{\prime}}\right\}, \\
X_{2,6}=Y_{2,6} \cup\left\{\mathbf{x}_{2^{\prime}, 3,6}, \mathbf{x}_{2,3,6^{\prime}}\right\}, & X_{3,4}=Y_{3,4} \cup\left\{\mathbf{x}_{3,4^{\prime}, 6}, \mathbf{x}_{3,4^{\prime}, 5}\right\}, \\
X_{3,5}=Y_{3,5} \cup\left\{\mathbf{x}_{3^{\prime}, 4,5}, \mathbf{x}_{3,4,5^{\prime}}\right\}, & X_{3,6}=Y_{3,6} \cup\left\{\mathbf{x}_{3^{\prime}, 4,6}, \mathbf{x}_{3,4,6^{\prime}}\right\}, \\
X_{4,5}=Y_{4,5} \cup\left\{\mathbf{x}_{1,4^{\prime}, 5}, \mathbf{x}_{1,4,5^{\prime}}\right\}, & X_{4,6}=Y_{4,6} \cup\left\{\mathbf{x}_{1,4^{\prime}, 6}, \mathbf{x}_{1,4,6^{\prime}}\right\} .
\end{array}
$$

It can be verified that

$$
\partial(O)=\bigcup X_{i, j}
$$

and $\delta^{O}(\mathbf{x}, \mathbf{y})<1$ whenever both $\mathbf{x}$ and $\mathbf{y}$ belong to the same $X_{i, j}$ (to verify $\delta^{C}(\mathbf{x}, \mathbf{y})<1$ for two points $\mathbf{x}$ and $\mathbf{y}$ it is convenient to find a point $\mathbf{z}$ such that both $\mathbf{z}+2(\mathbf{x}-\mathbf{z})$ and $\mathbf{z}+2(\mathbf{y}-\mathbf{z})$ belong to the interior of $C$, say $\operatorname{int}(C))$. Therefore, by Lemma 1 one obtains

$$
N(O) \leq 18 \text {. }
$$

Consequently, (1) and (2) together yield

$$
N(O)=N^{*}(O)=18 .
$$

Theorem 1 is proved.

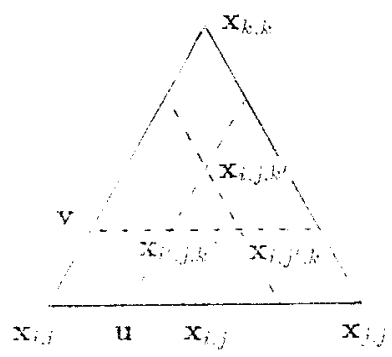

Fig. 2 


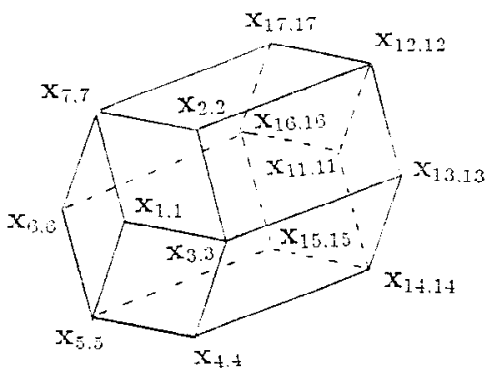

Fig. 3

\section{Rhombic Dodecahedra}

Theorem 2. Let $P_{1}$ be a rhombic dodecahedron (see Fig. 3), then

$$
N\left(P_{1}\right)=N^{*}\left(P_{1}\right)=18 .
$$

In addition, the kissing configuration, in which $N\left(P_{1}\right)=18$ can be attained, is unique.

Proof. Let $\mathbf{y}_{i, i}=\frac{1}{2} \mathbf{x}_{i, i}$ and denote the midpoint of $\mathbf{x}_{i, i} \mathbf{x}_{j, j}$ by $\mathbf{x}_{i, j}$. First, it is easy to see that $\left(\mathbf{y}_{1,1}+\frac{1}{2} P_{1}\right) \cap \partial\left(P_{1}\right)$ and $\left(\mathbf{y}_{2,2}+\frac{1}{2} P_{1}\right) \cap \partial\left(P_{1}\right)$ together can be divided into six parts $X_{21}, X_{22}, \ldots, X_{26}$ such that

$$
\delta^{P_{1}}(\mathbf{x}, \mathbf{y})<1
$$

whenever both $\mathbf{x}$ and $\mathbf{y}$ belong to the same part.

Writing

$$
Y_{i}=\operatorname{rint}\left(\left(\mathbf{y}_{i, i}+\frac{1}{2} P_{1}\right) \cap \partial\left(P_{1}\right)\right)
$$

and defining

$$
\begin{aligned}
X_{2} & =Y_{2} \cup\left(\mathbf{x}_{2,1} \mathbf{x}_{2,7} \mathbf{x}_{2,17} \mathbf{x}_{2,12}\right), \\
X_{3} & =Y_{3} \cup\left(\mathbf{x}_{3,7} \mathbf{x}_{3,2} \mathbf{x}_{3,12}\right) \cup\left(\mathbf{x}_{3,12} \mathbf{x}_{3,13} \mathbf{x}_{3,14}\right), \\
X_{4} & =Y_{4} \cup\left(\mathbf{x}_{4,1} \mathbf{x}_{4,3} \mathbf{x}_{4,13} \mathbf{x}_{4,14}\right), \\
X_{5} & =Y_{5} \cup\left(\mathbf{x}_{5,3} \mathbf{x}_{5,4} \mathbf{x}_{5,14}\right) \cup\left(\mathbf{x}_{5,14} \mathbf{x}_{5,15} \mathbf{x}_{5,16}\right), \\
X_{6} & =Y_{6} \cup\left(\mathbf{x}_{6,1} \mathbf{x}_{6,5} \mathbf{x}_{6,15} \mathbf{x}_{6,16}\right), \\
X_{7} & =Y_{7} \cup\left(\mathbf{x}_{7,5} \mathbf{x}_{7,6} \mathbf{x}_{7,16}\right) \cup\left(\mathbf{x}_{7,16} \mathbf{x}_{7,17} \mathbf{x}_{7,12}\right), \\
X_{12} & =Y_{12} \cup\left(\mathbf{x}_{12,16} \mathbf{x}_{12,17} \mathbf{x}_{12,7}\right) \cup\left(\mathbf{x}_{12,7} \mathbf{x}_{12,2} \mathbf{x}_{12,3}\right), \\
X_{13} & =Y_{13} \cup\left(\mathbf{x}_{13,11} \mathbf{x}_{13,12} \mathbf{x}_{13,2} \mathbf{x}_{13,3}\right), \\
X_{14} & =Y_{14} \cup\left(\mathbf{x}_{14,12} \mathbf{x}_{14,13} \mathbf{x}_{14,3}\right) \cup\left(\mathbf{x}_{14,3} \mathbf{x}_{14,4} \mathbf{x}_{14,5}\right), \\
X_{15} & =Y_{15} \cup\left(\mathbf{x}_{15,11} \mathbf{x}_{15,14} \mathbf{x}_{15,4} \mathbf{x}_{15,5}\right), \\
X_{16} & =Y_{16} \cup\left(\mathbf{x}_{16,14} \mathbf{x}_{16,15} \mathbf{x}_{16,5}\right) \cup\left(\mathbf{x}_{16,5} \mathbf{x}_{16,6} \mathbf{x}_{16,7}\right), \\
X_{17} & =Y_{17} \cup\left(\mathbf{x}_{17,11} \mathbf{x}_{17,16} \mathbf{x}_{17,6} \mathbf{x}_{17,7}\right),
\end{aligned}
$$


it can be verified that

$$
\delta^{P_{1}}(\mathbf{x}, \mathbf{y})<1, \quad \mathbf{x}, \mathbf{y} \in X_{i}
$$

and

$$
\partial\left(P_{1}\right)=\bigcup_{i=1}^{26} X_{i}
$$

(there are intervals among the indices). So, by Lemma 1 we have $N\left(P_{1}\right) \leq 18$. On the other hand, by suitable construction we get $N^{*}\left(P_{1}\right) \geq 18$ and therefore

$$
N\left(P_{1}\right)=N^{*}\left(P_{1}\right)=18 \text {. }
$$

Now, we proceed to show the uniqueness. Let $Z=\left\{\mathbf{z}_{1}, \mathbf{z}_{2}, \ldots, \mathbf{z}_{18}\right\}$ be a set of points such that $P_{1}+\{\mathbf{o}\} \cup\{2 Z\}$ is a kissing configuration. By Lemma 1 , without loss of generality, one has $\mathbf{z}_{1}=\mathbf{x}_{1,2}, \mathbf{z}_{2}=\mathbf{x}_{1,4}$ and $\mathbf{z}_{3}=\mathbf{x}_{1,6}$, or $\mathbf{z}_{1}=\mathbf{x}_{1,3}, \mathbf{z}_{2}=\mathbf{x}_{1,5}$ and $\mathbf{z}_{3}=\mathbf{x}_{1,7}$. Similarly, $\mathbf{z}_{16}=\mathbf{x}_{11,12}, \mathbf{z}_{17}=\mathbf{x}_{11,14}$ and $\mathbf{z}_{18}=\mathbf{x}_{11,16}$, or $\mathbf{z}_{16}=\mathbf{x}_{11,13}$, $\mathbf{z}_{17}=\mathbf{x}_{11,15}$ and $\mathbf{z}_{18}=\mathbf{x}_{11,17}$. Then, by considering four possibilities, finally we get

$$
\begin{aligned}
Z= & \left\{\mathbf{x}_{1,2}, \mathbf{x}_{1,4}, \mathbf{x}_{1,6}, \mathbf{x}_{3,3}, \mathbf{x}_{5,5}, \mathbf{x}_{7,7}, \mathbf{x}_{2,13}, \mathbf{x}_{3,14}, \mathbf{x}_{4,15},\right. \\
& \left.\mathbf{x}_{5,16}, \mathbf{x}_{6,17}, \mathbf{x}_{7,12}, \mathbf{x}_{12,12}, \mathbf{x}_{14,14}, \mathbf{x}_{16,16}, \mathbf{x}_{11,13}, \mathbf{x}_{11,15}, \mathbf{x}_{11,17}\right\},
\end{aligned}
$$

which implies the uniqueness. Theorem 2 is proved.

\section{Elongated Octahedra}

Theorem 3. Let $P_{2}$ be an elongated octahedron (see Fig. 4), then

$$
N\left(P_{2}\right) \leq 18
$$

More precisely, let

$$
\left\|\mathbf{x}_{8,8} \mathbf{x}_{18,18}\right\|=(1-\alpha)\left\|\mathbf{x}_{1,1} \mathbf{x}_{11,11}\right\|
$$

with a suitable number $\alpha$. It is easy to see that $0<\alpha<\frac{1}{2}$.

1. When $0<\alpha \leq \frac{1}{6}, N\left(P_{2}\right)=N^{*}\left(P_{2}\right)=18$.

2. When $\frac{1}{6}<\alpha \leq \frac{1}{4}, N\left(P_{2}\right)=18$ and $16 \leq N^{*}\left(P_{2}\right) \leq 18$.

Proof. Let $\mathbf{x}_{i, j}$ be the midpoint of $\mathbf{x}_{i, i} \mathbf{x}_{j, j}$. Writing $\mathbf{y}_{i, i}=\frac{1}{2} \mathbf{x}_{i, i}$ and

$$
Y_{i}=\operatorname{rint}\left(\left(\mathbf{y}_{i, i}+\frac{1}{2} P_{2}\right) \cap \partial\left(P_{2}\right)\right),
$$

and then defining

$$
\begin{aligned}
X_{1} & =Y_{1} \cup\left(\mathbf{x}_{1,3} \mathbf{x}_{1,2} \mathbf{x}_{1,9} \mathbf{x}_{1,8} \mathbf{x}_{1,7}\right), \\
X_{2} & =Y_{2} \cup\left(\mathbf{x}_{2,8} \mathbf{x}_{2,9}\right] \cup\left\{\mathbf{x}_{2,12}\right\}, \\
X_{12} & =Y_{12} \cup\left[\mathbf{x}_{12,19} \mathbf{x}_{12,18} \mathbf{x}_{12,11} \mathbf{x}_{12,14}\right),
\end{aligned}
$$




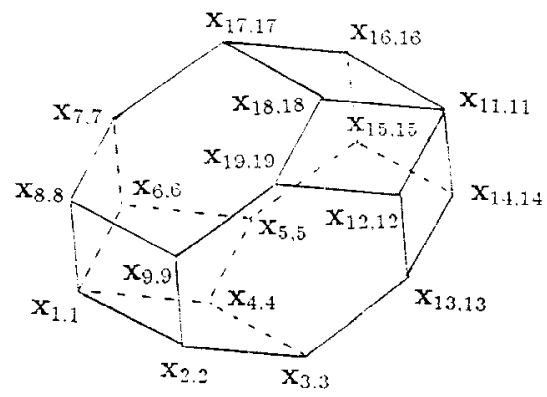

Fig. 4

$$
\begin{aligned}
X_{11} & =Y_{11} \cup\left(\mathbf{x}_{11,13} \mathbf{x}_{11,14} \mathbf{x}_{11,15} \mathbf{x}_{11,16} \mathbf{x}_{11,17}\right), \\
X_{16} & =Y_{16} \cup\left(\mathbf{x}_{16,14} \mathbf{x}_{16,15}\right] \cup\left\{\mathbf{x}_{16,6}\right\} \\
X_{6} & =Y_{6} \cup\left[\mathbf{x}_{6,5} \mathbf{x}_{6,4} \mathbf{x}_{6,1} \mathbf{x}_{6,8}\right), \\
X_{18} & =Y_{18} \cup\left[\mathbf{x}_{18,17} \mathbf{x}_{18,16} \mathbf{x}_{18,11} \mathbf{x}_{18,12}\right), \\
X_{8} & =Y_{8} \cup\left[\mathbf{x}_{8,7} \mathbf{x}_{8,6}\right) \cup\left\{\mathbf{x}_{8,18}\right\}, \\
X_{14} & =Y_{14} \cup\left[\mathbf{x}_{14,13} \mathbf{x}_{14,12}\right) \cup\left\{\mathbf{x}_{14,4}\right\}, \\
X_{4} & =Y_{4} \cup\left[\mathbf{x}_{4,3} \mathbf{x}_{4,2} \mathbf{x}_{4,1} \mathbf{x}_{4,6}\right), \\
X_{7} & =Y_{7} \cup\left[\mathbf{x}_{7,6} \mathbf{x}_{7,1}\right], \\
X_{17} & =Y_{17} \cup\left[\mathbf{x}_{17,16} \mathbf{x}_{17,11}\right) \cup\left\{\mathbf{x}_{17,7}\right\}, \\
X_{13} & =Y_{13} \cup\left[\mathbf{x}_{13,12} \mathbf{x}_{13,11}\right], \\
X_{3} & =Y_{3} \cup\left[\mathbf{x}_{3,2} \mathbf{x}_{3,1}\right) \cup\left\{\mathbf{x}_{3,13}\right\}, \\
X_{9} & =Y_{9} \cup\left[\mathbf{x}_{9,8} \mathbf{x}_{9,1}\right) \cup\left\{\mathbf{x}_{9,19}\right\}, \\
X_{19} & =Y_{19} \cup\left[\mathbf{x}_{19,18} \mathbf{x}_{19,11}\right), \\
X_{5} & =Y_{5} \cup\left[\mathbf{x}_{5,4} \mathbf{x}_{5,1}\right) \cup\left\{\mathbf{x}_{5,15}\right\}, \\
X_{15} & =Y_{15} \cup\left[\mathbf{x}_{15,14} \mathbf{x}_{15,11}\right),
\end{aligned}
$$

it can be verified that

$$
\delta^{P_{2}}(\mathbf{x}, \mathbf{y})<1, \quad \mathbf{x}, \mathbf{y} \in X_{i}
$$

and

$$
\partial\left(P_{2}\right)=\bigcup_{i=1}^{19} X_{i}
$$

(10 is not among the indices). Then it follows from Lemma 1 that

$$
N\left(P_{2}\right) \leq 18
$$

On the other hand, by simple constructions one can get

$$
N\left(P_{2}\right) \geq N^{*}\left(P_{2}\right) \geq 18
$$


in the first case, and

$$
N\left(P_{2}\right) \geq 18 \quad \text { and } \quad N^{*}\left(P_{2}\right) \geq 16
$$

in the second case (the parameter $\alpha$ plays important roles in the constructions). Thus, Theorem 3 follows.

Remark 1. It seems that $N^{*}\left(P_{2}\right)=16$ in the second case. If so, we will get another class of convex bodies such that $N(K) \neq N^{*}(K)$ (see [7]).

Remark 2. Let $P$ be an octahedron, a rhombic dodecahedron or an elongated octahedron of the first case of Theorem 3, the lattice kissing number of $P$ cannot be realized by its densest lattice packing. Similar phenomenon occurs at tetrahedron (see [8] and [9]).

\section{4. $n$-Dimensional Superballs}

Let $\alpha \geq 1$ and denote by $B_{\alpha}$ the superball

$$
\left\{\mathbf{x}=\left(x^{1}, x^{2}, \ldots, x^{n}\right) \in R^{n}:\left(\sum_{k=1}^{n}\left|x^{k}\right|^{\alpha}\right)^{1 / \alpha} \leq 1\right\} .
$$

It is easy to see that $B_{1}$ is a cross-polytope, $B_{2}$ is a ball, $B_{\infty}$ is a cube and the Minkowskimetric given by $B_{\alpha}$ can be represented as

$$
\delta^{B_{\alpha}}(\mathbf{x}, \mathbf{y})=\left(\sum_{k=1}^{n}\left|x^{k}-y^{k}\right|^{\alpha}\right)^{1 / \alpha} .
$$

Now we introduce a general lemma.

Lemma 2. The translative kissing number of $C$ is the maximal number of points $\mathbf{x}_{i} \in$ $\partial(C)$ such that

$$
\delta^{C}\left(\mathbf{x}_{i}, \mathbf{x}_{j}\right) \geq 1, \quad i \neq j
$$

This lemma follows directly from the fact that

$$
(\operatorname{int}(C)+\mathbf{x}) \cap(\operatorname{int}(C)+\mathbf{y})=\emptyset
$$

if and only if

$$
\delta^{C}(\mathbf{x}, \mathbf{y}) \geq 2 .
$$

By this lemma, a lower bound for $N\left(B_{\alpha}\right)$ can be achieved in a combinatorical way.

Lemma 3. Let $m \leq n$ be a positive integer and let $X$ be a set of points $\mathbf{x}_{i}=$ $\left(x_{i}^{1}, x_{i}^{2}, \ldots, x_{i}^{n}\right)$ with $x_{i}^{k}=0$ or $\pm 1, \sum_{k=1}^{n}\left|x_{i}^{k}\right|=m$ and

$$
\sum_{k=1}^{n}\left|x_{i}^{k}-x_{j}^{k}\right|^{\alpha} \geq m, \quad i \neq j \text {. }
$$


Then

$$
N\left(B_{\alpha}\right) \geq \operatorname{card}\{X\}
$$

Proof. Taking

$$
Y=\left\{\mathbf{y}_{i}=\frac{1}{m^{1 / \alpha}} \mathbf{x}_{i}: \mathbf{x}_{i} \in X\right\}
$$

we have

$$
\left(\sum_{k=1}^{n}\left|y_{i}^{k}\right|^{\alpha}\right)^{1 / \alpha}=\left(\frac{1}{m} \sum_{k=1}^{n}\left|x_{i}^{k}\right|^{\alpha}\right)^{1 / \alpha}=\left(\frac{1}{m} \sum_{k=1}^{n}\left|x_{i}^{k}\right|\right)^{1 / \alpha}=1
$$

which implies $\mathbf{y}_{i} \in \partial\left(B_{\alpha}\right)$. On the other hands, by (3) and (4),

$$
\delta^{B_{\alpha}}\left(\mathbf{y}_{i}, \mathbf{y}_{j}\right)=\left(\sum_{k=1}^{n} \frac{1}{m}\left|x_{i}^{k}-x_{j}^{k}\right|^{\alpha}\right)^{1 / \alpha} \geq 1, \quad i \neq j
$$

Therefore, by Lemma 2,

$$
N\left(B_{\alpha}\right) \geq \operatorname{card}\{Y\}=\operatorname{card}\{X\}
$$

which proves Lemma 3.

Let $f(n, m, \alpha)$ be the maximal possible card $\{X\}$, where $X$ is defined in Lemma 3, and write

$$
f(n, \alpha)=\max _{1 \leq m \leq n}\{f(n, m, \alpha)\}
$$

Then

$$
N\left(B_{\alpha}\right) \geq f(n, \alpha) .
$$

To get a lower bound for $f(n, \alpha)$, we have

\section{Lemma 4.}

$$
f(n, \alpha) \geq\left(\frac{9}{8}\right)^{(1-o(1)) n} \geq 3^{(0.1072-o(1)) n} .
$$

Proof. Since $\alpha \geq 1$, it is easy to see that

$$
\sum_{k=1}^{n}\left|x_{i}^{k}-x_{j}^{k}\right|^{\alpha} \geq \sum_{k=1}^{n}\left|x_{i}^{k}-x_{j}^{k}\right|
$$

holds for any two points $\mathbf{x}_{i}$ and $\mathbf{x}_{j}$ with integer coordinates. Therefore, it can be deduced that

$$
f(n, m, \alpha) \geq f(n, m, 1)
$$


and

$$
f(n, \alpha) \geq f(n, 1) .
$$

Writing

$$
Y=\left\{\left(x^{1}, x^{2}, \ldots, x^{n}\right): x^{k}=0 \text { or } \pm 1, \sum_{k=1}^{n}\left|x^{k}\right|=m\right\},
$$

we have

$$
\operatorname{card}\{Y\}=\left(\begin{array}{l}
n \\
m
\end{array}\right) 2^{m} .
$$

For any point $\mathbf{x}_{i} \in Y$, by easy computation, there are at most

$$
g(n, m, 1)=\left(\begin{array}{c}
m \\
h(m)
\end{array}\right)\left(\begin{array}{c}
n-h(m) \\
m-h(m)
\end{array}\right) 2^{m-h(m)}
$$

points $\mathbf{x}_{j} \in Y$ such that

$$
\sum_{k=1}^{n}\left|x_{j}^{k}-x_{i}^{k}\right|<m
$$

where $h(m)=[m / 2]+1$. Hence, by (8) and (9),

$$
\left(\begin{array}{l}
n \\
m
\end{array}\right) 2^{m}-f(n, m, 1) g(n, m, 1) \leq 0
$$

and therefore

$$
f(n, m, 1) \geq \frac{\left(\begin{array}{c}
n \\
m
\end{array}\right) 2^{m}}{g(n, m, 1)}=2^{h(m)}\left(\begin{array}{c}
n \\
m
\end{array}\right)\left(\begin{array}{c}
m \\
h(m)
\end{array}\right)^{-1}\left(\begin{array}{c}
n-h(m) \\
m-h(m)
\end{array}\right)^{-1} .
$$

Writing $n / m=l$, by Stirling Formula and detailed computation, we have

$$
f\left(n, \frac{n}{l}, 1\right) \geq\left(2^{1-3 / 2 l}(2 l-1)^{1 / 2 l-1} l\right)^{(1-o(1)) n} .
$$

By basic analysis, it can be shown that the function

$$
f(x)=2^{1-3 / 2 x}(2 x-1)^{1 / 2 x-1} x
$$

attains its maximum $\frac{9}{8}$ at $x=\frac{9}{2}$. Therefore, by (5) and (7),

$$
f(n, \alpha) \geq\left(\frac{9}{8}\right)^{(1-o(1)) n} \geq 3^{(0.1072-o(1)) n} .
$$

Lemma 4 is proved.

It is well known that

$$
N^{*}(K) \leq N(K) \leq 3^{n}-1
$$

for every $n$-dimensional convex body (see [4] and [6]). Therefore, by (6) and Lemma 4, 
we have

Theorem 4.

$$
3^{(0.1072-o(1)) n} \leq N\left(B_{\alpha}\right) \leq 3^{n} .
$$

Remark 3. According to the referee, I. Talata recently obtained an exponential lower bound for $N(K)$ for general $n$-dimensional convex bodies $K$.

\section{Acknowledgments}

For helpful discussion we are obliged to Professor C. A. Rogers. For helpful comments we are obliged to the referee.

\section{References}

1. J.H. Conway and N.J.A. Sloane, Sphere Packings, Lattices and Groups, Springer-Verlag, New York, 1988.

2. G. Fejes Tóth and W. Kuperberg, Packing and covering with convex sets, in Handbook of Convex Geometry (P.M. Gruber and J.M. Wills, eds.), pp. 799-860, North-Holland, Amsterdam, 1993.

3. P.M. Gruber and C.G. Lekkerkerker, Geometry of Numbers (2nd edn.), North-Holland, Amsterdam, 1987.

4. H. Hadwiger, Über Treffanzahlen bei translationsgleichen Eikörpern, Arch. Math. 8 (1957), 212-213.

5. K. Leichtweiß, Konvexe Mengen, Springer-Verlag, Berlin, 1980.

6. H. Minkowski, Geometrie der Zahlen, Leipzig, Berlin, 1896, 1910; New York, Chelsea, 1953.

7. C. Zong, An example concerning translative kissing numbers of a convex body, Discrete Comput. Geom. 12 (1994), 183-188.

8. C. Zong, The kissing numbers of tetrahedra, Discrete Comput. Geom. 15 (1996), 239-252.

9. C. Zong, Strange Phenomena in Convex and Discrete Geometry, Springer-Verlag, New York, 1996.

10. C. Zong, The translative kissing numbers of the Cartesian product of two convex bodies, one of which is two-dimensional, Geom. Dedicata 65 (1997), 135-145.

11. C. Zong, The kissing numbers of convex bodies—a brief survey, Bull. London Math. Soc. 30 (1998), 1-10.

Received December 24, 1996, and in revised form October 7, 1997. 